\title{
Utility and limitations of ejection fraction and of diastolic dysfunction in heart failure patients
}

\author{
Gian Francesco Mureddu', Andrea Faggiano \\ ${ }^{1}$ Cardiac Rehabilitation and Secondary Cardiovascular Prevention Unit, Cardiology 2 Unit, Department of Internal \\ Medicine, San Giovanni-Addolorata Hospital, Rome; ${ }^{2}$ Medical student, University of Milan, Italy
}

\begin{abstract}
The echocardiographic evaluation of left ventricular (LV) systolic function, and especially of ejection fraction (EF) plays a central role in the diagnosis of heart failure (HF) due to its undisputed prognostic value. Limitations of EF are substantially: i) the variability and reproducibility of measurements, and ii) the load-dependence. Measurement of stroke volume, longitudinal function and myocardial strain can overcome the limitations of EF in assessing the contractile reserve of patients with HF and may help to define both the phenotype and prognosis of the disease. The recognition of diastolic dysfunction (mainly by echocardiography) is the pathophysiological basis to make diagnosis of HF with preserved ejection fraction (HFpEF). The limitations are essentially related to its feasibility, since performing a multi-parametric quantitative echocardiographic evaluation, as indicated by the guidelines, may be difficult in clinical practice. Difficulties in method standardization, the poor attitude of cardiologists to test their reproducibility (test-retest, variability) favor the evaluation "at-a-glance" of LV structural and functional LV abnormalities.
\end{abstract}

\section{Left ventricular systolic function}

The echocardiographic assessment of left ventricular (LV) systolic function, especially of ejection fraction (EF) plays a central role in the diagnostic and prognostic assessment of patients with either suspected or ascertained heart failure (HF). This role, always accepted by clinicians, has been strongly strengthened by the guidelines of the European Society of Cardiology [1]. In fact, after the initial history and clinical evaluation and the electrocardiogram, which are used to select the patient, and after a possible evaluation with biomarkers (natriuretic peptides), mainly used as an exclusion test, echocardiography serves to confirm the diagnosis and to define the phenotype. If HF is confirmed, the next step is to determine the etiology and choose the appropriate therapy. EF also plays a central role in the therapeutic choice. As the 2016 European guidelines report, the main terminology used to describe HF is historical and is based on measurement of the EF.

The EF values are typically considered normal when $\geq 50 \%$. Therefore, in the presence of HF with normal EF the patient is classified as having HF with preserved $\mathrm{EF}$ (HFpEF), while patients with depressed EF (considered for $\mathrm{EF}<40 \%$ ) are classified as HF with reduced $\mathrm{EF}$ (HFrEF). Those patients who present with an EF between $40-49 \%$ represent a "new" category, considered as a grey area now defined as HF with mid-range EF (HFmrEF). The distinction of patients on the EF basis is important because the different phenotypes identified by the EF underlie various etiologies, demographic characteristics, comorbidities and response to therapies [2]. Actually, a similar classification was already proposed previously by the Echocardiographic Heart of England Screening Study (ECHOES) [3], a population screening conducted on 6162 random selected patients, showing that borderline systolic dysfunction (EF: $40-50 \%$ ) was associated with an adverse prognosis. However, the $40 \%$ cut-off used for the diagnosis of HFpEF form is arbitrary and many patients with mid-range EF have been classified as patients with HFpEF in clinical trials $[1,4]$.

\section{Usefulness and limitations}

The usefulness of EF in HF patients is undisputed as it is one of the most important predictors of mortality in HF of any etiology [1,5-7], mainly of ischemic origin, as an expression of post-infarct ventricular remodeling [8-10]. Moreover, EF has a pivotal role in the indication of drug therapy, which in turn affects the long-term prognosis in both symptomatic and asymptomatic patients [1113]. The predictive prognostic power of EF has also been demon-

strated in studies of asymptomatic population [14] and, recently,
This article is distributed under the terms of the Creative Commons Attribution Noncommercial License (by-nc 4.0) which permits any noncommercial use, distribution, and reproduction in any medium, provided the original author(s) and source are credited. 
was also confirmed in patients with acute ST elevation myocardial infarction (STEMI) treated with primary angioplasty.

In fact, in a cohort of 2086 consecutive STEMI patients, enrolled between 2007 and 2016, patients presenting with midrange $\mathrm{EF}(858,41 \%)$ had a worse long-term prognosis ( 8 years) than those who had a normal FE (1013, 48\%) (9.8\% vs 7.2\%, $\mathrm{p}<0.01)$ but showing a lower mortality rate compared to those with reduced $\mathrm{EF}(9.8 \%$ vs $29.2 \%, \mathrm{p}<0.001)$ [15]. It has also been shown that serial evaluation of EF changes over time is a powerful predictor of survival and hospitalization in $\mathrm{HF}$, thus representing a simple but feasible measure of prognosis in heart failure with HFrEF [16]. This result is in line with the results of a meta-analysis that included 30 mortality trials, 25 therapeutic interventions for a total of 69,766 patients and 88 studies that had LV remodeling in same therapies as End-points (19,921 patients). The risk of death (odds ratio) in mortality trials was correlated with the pharmacological effect on both EF $(r=0.51, p<0.001)$ and LV remodeling [17].

The main limitations of LV function evaluation by EF are substantially of two types i) the variability and reproducibility of the data, and ii) the load dependence of EF. Previous studies have reported a high intra-observer variability of both end-diastolic volume (EDV) and end-systolic volume (ESV) (11-15\% and 15-25\% respectively), and of EF (7-10\%), regardless of the methods used (Area-length, Simpson). This variability increases in dilated ventricles and for the lowest EF values $(<20 \%)$, markedly limiting the measurements reproducibility, at least in laboratories that have not performed intra and inter-observer variability tests. The second important limit lies the fact that the $\mathrm{EF}$ is a parameter of chamber function (expressing the emptying of the left ventricle) rather than a real index of LV contractile function. Thus, it is strongly affected by LV geometry and load. The relationship between EDV $\left(\mathrm{ml} / \mathrm{m}^{2}\right)$ and the EF is inverse and is regulated by LV geometry. In the initial phase of heart failure, LV pre-load and after-load tend to increase, acting in opposite directions on the EF which therefore remains normal despite the development of eccentric hypertrophy and dilatation, tending to stay stable for progressive volume increases as expressed by Starling principle [18]. For this reason, in advanced HF, EF can remain constant even in the presence of significant pre-load and after-load changes. On the contrary, in the presence of concentric geometry, EF can vary considerably with the loading conditions and may overestimate the real contractile ventricular performance. In fact, the contractile amplification towards the endocardium determined by the interaction that the myocardial fibers, structurally oriented in different ways (opposed helical, radial and longitudinal fibers), exercise during contraction can result in an amplification of the shortening measured at the endocardium and in a overestimation of EF. This phenomenon is particularly evident in concentric hypertrophy. In experimental models, a $15 \%$ shortening of the single isolated myocardial fiber correspond to a thickening of about $40 \%$ of the endocardium [19]. This explains why a measure of the contractile function taken at the center of the LV wall (midwall shortening) may reveal a subclinical latent contractile dysfunction earlier than those taken at endocardium (such as fractional shortening or EF). The relationship of midwall fractional shortening to circumferential end-systolic stress [20-22] (that physiologically oppose to the contraction) showed a significant prognostic value in different clinical settings.

Measurement of stroke volume, longitudinal function and measurements of myocardial strain may exceed the limitations of $\mathrm{EF}$ in assessing the contractile reserve of heart failure patients and may define the phenotype and prognosis [23] when considered together with morphologic LV data (chamber dilatation, wall thickness, relative wall thickness) [24].

\section{Left ventricular diastolic function}

The diagnosis of heart failure with preserved systolic function (HFpEF) can be difficult because it requires multi-parametric evaluation and can coexist with a number of comorbidities that makes that phenotype peculiar and complex. The physio-pathological basis needed for diagnosis of HFpEF is the recognition of diastolic dysfunction, which can also be associated with a preclinical systolic dysfunction, the latter being detectable through the assessment of myocardial deformation parameters or, as previously mentioned, the LV end-systolic stress/LV function relationships [21]. The diastolic dysfunction diagnosis should include at least the evaluation of the transmitral flow velocities (peak E velocity, E deceleration time, EA velocity ratio) and the tissue Doppler velocity (TDI) at the level of the lateral and medial mitral annulus $(E / e \geq 13 \mathrm{~cm} / \mathrm{sec}$ and mean e' septal and lateral wall $\geq 9$ ) [25]. An important finding of confirmation, necessary in case of initial suspicion/evidence of HFpEF / HFmrEF, consists in the co-existence of structural cardiac changes demonstration in addition to the functional ones, able to explain the clinical presentation of heart failure (HF). Among these, the ESC guidelines underline the importance of detecting LV hypertrophy (left ventricular mass index $\geq 115 \mathrm{~g} / \mathrm{m}^{2}$ for males and $\geq 95 \mathrm{~g} / \mathrm{m}^{2}$ for females) left atrial dilatation (left atrial volume index $>34 \mathrm{ml} / \mathrm{m}^{2}$ ) which both identify HF stage B and are obviously present in the more advanced stages (C and D). Diastolic dysfunction, including preclinical, asymptomatic or symptomatic forms, has a high negative prognostic value confirmed by population and clinical studies [26,27]. Clinical conditions associated with HFpEF [25] are advanced age, female gender, arterial hypertension, atrial fibrillation $(\mathrm{AF})$, renal dysfunction, obesity, metabolic syndrome, obstructive pulmonary disease (COPD) and/or pulmonary hypertension $(\mathrm{PH})$, sleep apnoea syndrome and physical deconditioning. Many of these conditions are included in a more extensive definition of HF stage B $[28,29]$. The diagnosis of HFpEF in patients with AF can be difficult. AF may be a consequence of HFpEF or simply a determinant of a transient decompensation condition, which however may underlie a predisposition to HF development (stage B). Therefore, the echocardiographic recognition of diastolic dysfunction is central in confirming diagnostic hypothesis of $\mathrm{HfpEF} /$ preclinical diastolic cardiac dysfunction (stage B of the HF). It is useful in subjects exposed to risk, mainly with advancing age because it is a condition with a high prevalence in the elderly $[30,31]$.

The limitations are essentially linked to the feasibility of performing a multi-parametric quantitative echocardiographic evaluation (to recognize structural and functional myocardial abnormalities during hospital admission or in the outpatient setting. The data from the VASTISSIMO study (EValuation of the AppropriateneSs of the preclInical phase (Stage A and Stage B) of Heart Health Management in Outpatient Clinics in Italy) indicate that this quantification, performed in the hospital and / or community practice, is still not optimal. The EF and the diastolic dysfunction are important predictors of clinical events and mortality in HF.

The limitations are essentially related to its feasibility, since performing a multi-parametric quantitative echocardiographic evaluation, as indicated by the guidelines, may be difficult in clinical practice. Difficulties in method standardization, the poor attitude of cardiologists to test their reproducibility (test-retest, variability) favor the evaluation "at-a-glance" of LV structural and functional LV abnormalities. In conclusion, although obtaining a complete LV systolic and diastolic function assessment may be considered to be difficult and time consuming, only maintaining an elevated skill in diagnostic techniques can guarantee to cardiolo- 
gists a progression in the knowledge and in the comprehension of such a complex clinical picture.

\section{References}

1. Ponikowski P, Voors AA, Anker SD, et al. 2016 ESC Guidelines for the diagnosis and treatment of acute and chronic heart failure: The Task Force for the diagnosis and treatment of acute and chronic heart failure of the European Society of Cardiology (ESC). Developed with the special contribution of the Heart Failure Association (HFA) of the ESC. Eur J Heart Fail 2016;18:891-975.

2. Butler J, Fonarow GC, Zile MR, et al. Developing therapies for heart failure with preserved ejection fraction: current state and future directions. JACC Heart Fail 2014;2:97-112.

3. Hobbs FDR, Roalfe AK, Davis RC, et al. Prognosis of allcause heart failure and borderline left ventricular systolic dysfunction: 5 year mortality follow-up of the Echocardiographic Heart of England Screening Study (ECHOES). Eur Heart J 2007;28:1128-34.

4. Kelly JP, Mentz RJ, Mebazaa A, et al. Patient selection in heart failure with preserved ejection fraction clinical trials. J Am Coll Cardiol 2015;65:1668-1682.

5. Writing Committee Members, Yancy CW, Jessup M, et al. 2016 ACC/AHA/HFSA Focused Update on New Pharmacological Therapy for Heart Failure: An Update of the 2013 ACCF/AHA Guideline for the Management of Heart Failure. A Report of the American College of Cardiology/American Heart Association Task Force on Clinical Practice Guidelines and the Heart Failure Society of America. Circulation 2016;134:e282-93. Erratum in: Correction to: 2016 ACC/AHA/HFSA Focused Update on New Pharmacological Therapy for Heart Failure: An Update of the 2013 ACCF/AHA Guideline for the Management of Heart Failure: A Report of the American College of Cardiology Foundation/American Heart Association Task Force on Clinical Practice Guidelines and the Heart Failure Society of America. Circulation 2016;134:e282-e93.

6. Ouwerkerk W, Voors AA, Zwinderman AH. Factors influencing the predictive power of models for predicting mortality and/or heart failure hospitalization in patients with heart failure. JACC Heart Fail 2014;2:429-36.

7. Rahimi K, Bennett D, Conrad N, et al. Risk prediction in patients with heart failure: a systematic review and analysis. JACC Heart Fail 2014;2:440-6.

8. Pfeffer MA, Braunwald E, Moye LA, et al. Effect of captopril on mortality and morbidity in patients with left ventricular dysfunction after myocardial infarction. Results of the survival and ventricular enlargement trial. The SAVE Investigators. N Engl J Med 1992;327:669-77.

9. Køber L, Torp-Pedersen C, Carlsen JE, et al. A clinical trial of the angiotensin-converting-enzyme inhibitor trandolapril in patients with left ventricular dysfunction after myocardial infarction. N Engl J Med 1995;333:1670-6.

10. Acute Infarction Ramipril Efficacy (AIRE) Study Investigators. Effect of ramipril on mortality and morbidity of survivors of acute myocardial infarction with clinical evidence of heart failure. Lancet 1993;342:821-8.

11. SOLVD Investigators. Effect of enalapril on survival in patients with reduced left ventricular ejection fractions and congestive heart failure. N Engl J Med 1991;325:293-302.

12. SOLVD Investigators, Yusuf S, Pitt B, et al. Effect of enalapril on mortality and the development of heart failure in asymptomatic patients with reduced left ventricular ejection fractions. N Engl J Med 1992;327:685-91.

13. Jong P, Yusuf S, Rousseau MF, et al. Effect of enalapril on 12year survival and life expectancy in patients with left ventricular systolic dysfunction: a follow-up study. Lancet 2003;361:1843-8.

14. Wang TJ, Evans JC, Benjamin EJ, et al. Natural history of asymptomatic left ventricular systolic dysfunction in the community. Circulation 2003;108:977-98.

15. Margolis G, Khoury S, Ben-Shoshan J, et al. Prognostic implications of mid-range left ventricular ejection fraction on patients presenting with ST-segment elevation myocardial infarction. Am J Cardiol 2017;120:186-90.

16. Breathett K, Allen LA, Udelson J, et al. Changes in left ventricular ejection fraction predict survival and hospitalization in heart failure with reduced ejection fraction. Circ Heart Fail 2016;9:e002962.

17. Kramer DG, Trikalinos TA, Kent DM, et al. Quantitative evaluation of drug or device effects on ventricular remodeling as predictors of therapeutic effects on mortality in patients with heart failure and reduced ejection fraction a meta-analytic approach. J Am Coll Cardiol 2010;56:392-406.

18. Gaasch WH. Diagnosis and treatment of heart failure based on left ventricular systolic or diastolic dysfunction. J Am Med Assoc 1994;271:1276-80.

19. de Simone G, Devereux RB. Rationale of echocardiographic assessment of left ventricular wall stress and midwall mechanics in hypertensive heart disease. Eur J Echocard 2002;3:192-8.

20. Ballo P, Barone D, Bocelli A, et al. Left ventricular longitudinal systolic dysfunction is an independent marker of cardiovascular risk in patients with hypertension. Am J Hypertens 2008;21:1047-54.

21. Borlaug BA, Lam CSP, Roger VL, et al. Contractility and ventricular systolic stiffening in hypertensive heart disease: insights into the pathogenesis of heart failure with preserved ejection fraction. J Am Coll Cardiol 2009;54:410-8.

22. De Simone G, Devereux RB, Koren MJ, et al. Midwall left ventricular mechanics. An independent predictor of cardiovascular risk in arterial hypertension. Circulation 1996;93:259-65.

23. De Marco M, Gerdts E, Mancusi C, et al. Influence of left ventricular stroke volume on incident heart failure in a population with preserved ejection fraction (From the Strong Heart Study). Am J Cardiol 2017;119:1047-52.

24. Shah SJ, Katz DH, Selvaraj S, et al. Phenomapping for novel classification of heart failure with preserved ejection fraction. Circulation 2015;131:269-79.

25. Ponikowski P, Voors AA, Anker SD, et al. 2016 ESC Guidelines for the diagnosis and treatment of acute and chronic heart failure - Web Addenda. Eur Heart J 2016;37:2129-2200.

26. Echouffo-Tcheugui JB, Erqou S, Butler J, et al. Assessing the risk of progression from asymptomatic left ventricular dysfunction to overt heart failure: A systematic overview and meta-analysis. JACC Heart Fail 2016;4:237-48.

27. Wan SH, Vogel MW, Chen HH. Pre-clinical diastolic dysfunction. J Am Coll Cardiol 2014;63:407-16.

28. Mureddu GF, Nistri S, Faggiano P, et al. Evaluation of the appropriateness of the preclinical phase (stage A and stage B) of heart failure Management in Outpatient clinics in Italy rationale and design of the 'VASTISSIMO' study. J Cardiovasc Med (Hagerstown) 2016;17:501-9.

29. Schocken DD, Benjamin EJ, Fonarow GC, et al. Prevention of heart failure: a scientific statement from the American Heart Association Councils on epidemiology and prevention, clinical 
cardiology, cardiovascular nursing, and high blood pressure research; Quality of Care and Outcomes Research Interdisciplinary Working Group; and Functional Genomics and Translational Biology Interdisciplinary Working Group. Circulation 2008;117:2544-65.

30. Mureddu GF, Agabiti N, Rizzello V, et al. Prevalence of preclinical and clinical heart failure in the elderly. A popula- tion-based study in Central Italy. Eur J Heart Fail 2012;14: 718-29.

31. Mureddu GF, Tarantini L, Agabiti N, et al. Evaluation of different strategies for identifying asymptomatic left ventricular dysfunction and pre-clinical (stage B) heart failure in the elderly. Results from 'PREDICTOR', a population based-study in central Italy. Eur J Heart Fail 2013;15:1102-12. 